\section{Geminin is essential to prevent endoreduplication and to form pluripotent cells during mammalian development}

Michael A. Gonzalez, ${ }^{1,4,5}$ Kiku-e K. Tachibana, ${ }^{1,4}$ David J. Adams, ${ }^{2,4}$ Louise van der Weyden, ${ }^{2}$ Myriam Hemberger, ${ }^{3}$ Nicholas Coleman, ${ }^{1}$ Allan Bradley, ${ }^{2}$ and Ronald A. Laskey ${ }^{1}$

${ }^{1}$ Medical Research Council Cancer Cell Unit, Hutchison/MRC Research Centre, Cambridge CB2 2XZ, UK; ${ }^{2}$ Wellcome Trust Sanger Institute, Hinxton, Cambridge CB10 1SA, UK; ${ }^{3}$ The Babraham Institute, Cambridge CB2 4AT, UK

In multicellular eukaryotes, geminin prevents overreplication of DNA in proliferating cells. Here, we show that genetic ablation of geminin in the mouse prevents formation of inner cell mass (ICM) and causes premature endoreduplication at eight cells, rather than 32 cells. All cells in geminin-deficient embryos commit to the trophoblast cell lineage and consist of trophoblast giant cells (TGCs) only. Geminin is also down-regulated in TGCs of wild-type blastocysts during $S$ and gap-like phases by proteasome-mediated degradation, suggesting that loss of geminin is part of the mechanism regulating endoreduplication.

Supplemental material is available at http://www.genesdev.org

Received January 12, 2006; revised version accepted May 15, 2006.

Mammalian embryonic development begins with a series of cleavage divisions commencing in the fertilized egg. Two distinct cell types are generated during the first events of cellular differentiation and determination of lineage, giving rise to the inner cell mass (ICM) and trophectoderm of the blastocyst. The ICM develops into the embryo proper whereas all extraembryonic tissues are derived from the surrounding trophectoderm (Ralston and Rossant 2005).

Cells in the developing mammalian embryo have unique cell cycle characteristics that remain poorly understood. In embryonic stem (ES) cell lines derived from explant cultures of ICM, more than two-thirds of the mitotic ES cell cycle is devoted to $S$ phase, and the gap phases (G1 and G2) are shorter than those of their somatic counterparts. Cyclin-dependent kinase (cdk) activity remains high throughout the ES cell cycle and oscillations are only observed with the mitotic cyclin, cyclin

[Keywords: Geminin; endoreduplication; trophoblast; Oct4; Cdx2; pluripotency]

${ }^{4}$ These authors contributed equally to this work.

${ }^{5}$ Corresponding author.

E-MAIL mg322@hutchison-mrc.cam.ac.uk; FAX 44-1223-763293.

Article is online at http://www.genesdev.org/cgi/doi/10.1101/gad.379706.
B1 (Stead et al. 2002). Cells of the ICM retain a diploid DNA content, but interestingly cells in the trophectoderm can acquire a $>1000 \mathrm{C}$ DNA content. The latter form trophoblast giant cells (TGCs) by undergoing endoreduplication, in which $S$ phases alternate with "gaplike" phases, bypassing mitosis. Cyclin E is required for endoreduplication in mouse TGCs of the trophectoderm (Geng et al. 2003; Parisi et al. 2003).

Although a transcriptional program principally driven by Oct4, Cdx2, Nanog, and Gata6 regulates cell identity in the mammalian embryo, the precise control by which cell proliferation is coordinated with differentiation and determination of cell lineage has not been established (Ralston and Rossant 2005). In this study, we have used a genetic approach to investigate the role of geminin during mammalian development. Geminin inhibits prereplication complex (pre-RC) assembly in somatic cells by preventing Cdt1 from recruiting minichromosome maintenance (MCM) proteins to chromatin, from $S$ phase to the metaphase:anaphase transition, when it is targeted for degradation by the anaphase-promoting complex/cyclosome (APC/C) (McGarry and Kirschner 1998; Wohlschlegel et al. 2000; Gonzalez et al. 2004). Moreover, geminin controls differentiation as well as cell proliferation during late metazoan development. Geminin inhibits both Six3 and Hox transcription factors in medaka retina and the mouse embryo, respectively, and maintains an undifferentiated state in neural progenitor cells of Xenopus laevis by antagonising Brg-1 activity (Del Bene et al. 2004; Luo et al. 2004; Seo et al. 2005). Here, we show that geminin ablation in the mouse embryo causes premature endoreduplication at eight cells and that all eight cells express trophoblast markers and fail to express markers of pluripotency.

\section{Results and Discussion}

A murine ES cell line in which one geminin allele had been disrupted by a trap insertion event was obtained from the Sanger Institute Gene Trap Resource (SIGTR) (Supplementary Figs. S1A-D, S2; Skarnes et al. 2004). Analysis of the gene trap genome junction and the chimaeric transcript generated by splicing of geminin to the $\beta$-geo gene trap cassette indicated that the first intron of geminin had been trapped. The geminin allele (Gmnn ${ }^{\mathrm{GT}}$ ) was transmitted through the germline using standard procedures. Gmnn ${ }^{+\mathrm{GT}}$ mice showed no overt abnormalities.

To determine if geminin is essential for mouse development, test-matings between $\mathrm{Gmnn}^{+/ \mathrm{GT}}$ mice were established. No homozygous mutant $\left(G m n n^{\mathrm{GT} / \mathrm{GT}}\right)$ mice were detected in the resultant progeny $(n=123)$. To determine the stage and cause of embryonic lethality, mouse embryos were recovered and genotyped at embryonic days 15 (E15) and E8.5-E9.5. Homozygous Gmnn GT/GT embryos were not detected at any of these post-implantation stages. The absence of resorbed embryos or empty deciduas suggested that loss of geminin results in preimplantation lethality and that geminin is required for early embryogenesis.

To confirm this, preimplantation mouse embryos from $\mathrm{Gmnn}^{+/ \mathrm{GT}}$ intercrosses were examined by light microscopy at E3.5. Seventy embryos from a total of 296 were morphologically abnormal (mutant) and consisted 
of only eight cells (Supplementary Fig. S3) whereas the other 226 embryos had developed into blastocysts consisting of at least 32 cells $\left(\chi^{2}=0.29, \mathrm{df}=1, p=0.59\right)$. The nuclear morphology and DNA content of the mutant eight-cell arrested embryos $(n=48)$ were analyzed by confocal microscopy using the fluorescent nuclear stain DAPI. The nuclei of the mutant eight-cell arrested embryos were grossly enlarged compared with nuclei from eight-cell wild-type embryos (E2.5) and cells of the E3.5 blastocyst ICM (Fig. 1A). The DNA content was assessed semiquantitatively as a function of nuclear volume and DAPI fluorescence intensity of individual nuclei from mutant and wild-type embryos (Fig. 1B) using Volocity LE 3.0.2 software (http://mac.softpedia.com). All intact nuclei from mutant eight-cell arrested embryos contained at least three times more DNA than cells from wild-type blastocysts. Notably, nuclei from mutant eight-cell arrested embryos also contained more DNA than wild-type progenitor TGCs at E3.5, suggesting that endoreduplication had started early in the mutant embryos. It is unlikely that any of the $G m n n^{\mathrm{GT} / \mathrm{GT}}$ embryos were normal at this stage, as none were recovered postimplantation.

To confirm that the eight-cell arrested mouse embryos were homozygous for the disrupted geminin gene and therefore lack geminin protein, we used immunofluorescent staining of geminin. Thirty-three of 33 morphologically abnormal eight-cell embryos contained no detectable geminin whereas geminin could be detected in blastocysts recovered from the same matings $(n=82)$ (Fig. 2B). We also compared the eight-cell arrested Gmnn ${ }^{\mathrm{GT} / \mathrm{GT}}$ embryos (E3.5) to eight-cell wild-type embryos (E2.5). Since geminin protein is clearly present in eight-cell wild-type embryos but not in eight-cell arrested embryos, the latter correspond to the Gmnn GT/GT embryos. Geminin knockdown causes an accumulation of a $>4 \mathrm{C}$ DNA content without entry into mitosis in some human cancer cell lines but not in others (Melix-

A
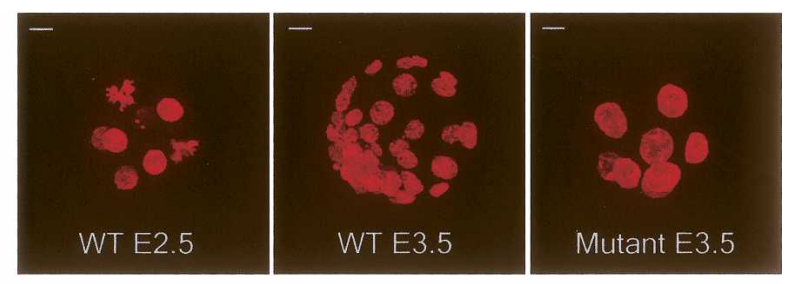

B
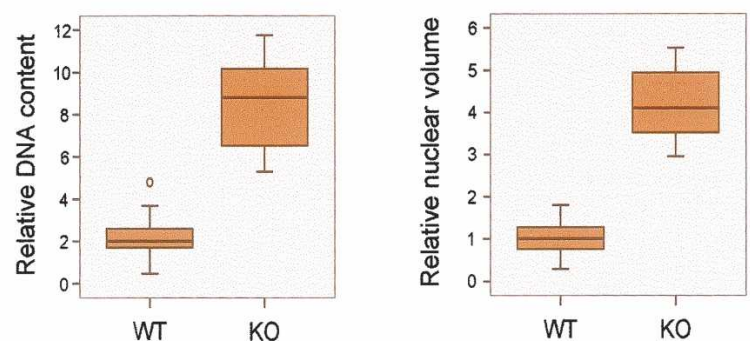

Figure 1. Ablation of geminin results in eight-cell arrested mouse embryos that resemble TGCs in nuclear size and DNA content. $(A)$ Z stacks were collected throughout DAPI-stained embryos and compiled into a single image. Bar, $10 \mu \mathrm{m}$. (B) Box plots showing relative DNA content analysis of wild-type E3.5 nuclei and nuclei from eight-cell arrested embryos resulting from $\mathrm{Gmnn}{ }^{\mathrm{GT} /+}$ intercrosses. (Line) Median; (box) interquartile range; (whiskers) expected range; (circle) extreme value.

\section{A}
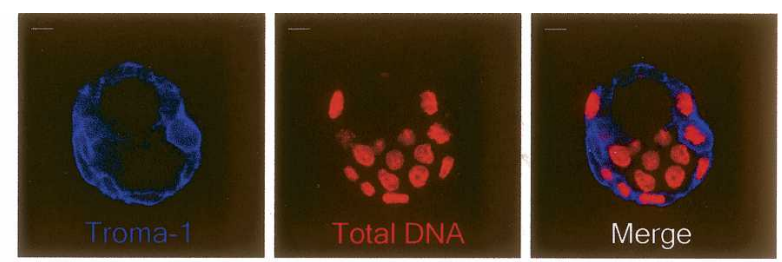

B
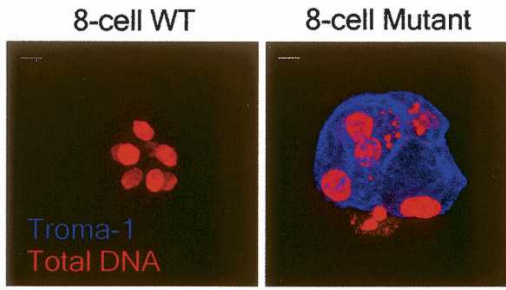

32-cell WT
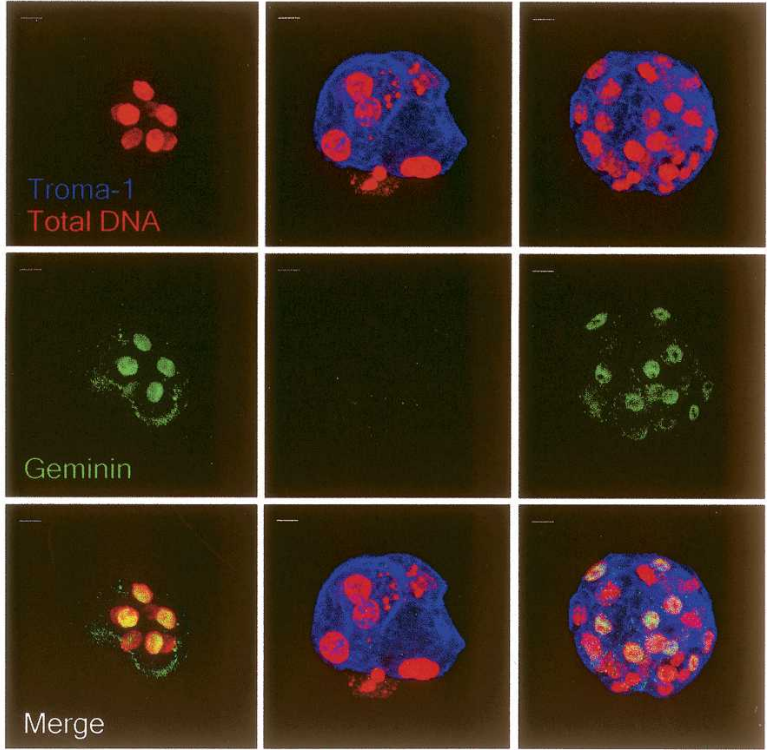

Figure 2. Geminin-null embryos consist of TGCs only. $(A)$ Troma-1 (blue) is a trophoblast-specific marker and is absent in cells of the ICM. Total DNA (red). (B) Troma-1 (blue) is expressed in geminin-null embryos but not wild-type eight-cell embryos. Geminin (green) is present in eight-cell and $>32$-cell wild-type mouse embryos. The trophectoderm is well established by the $>32$-cell stage. Bar, $10 \mu \mathrm{m}$.

etian et al. 2004; Zhu et al. 2004), suggesting that a redundant pathway is active in the latter (Tachibana and Nigg 2006). We demonstrate here that genetic ablation of geminin in the mouse also results in acquisition of a $>4 \mathrm{C}$ DNA content and concomitant absence of mitotic ICM cells. Thus, we conclude that geminin is essential for preventing repeated cycles of DNA replication during early mammalian development.

Since the phenotype of Gmnn ${ }^{\mathrm{GT} / \mathrm{GT}}$ cells was reminiscent of TGCs at the late blastocyst stage, we asked whether all cells in Gmnn ${ }^{\mathrm{GT} / \mathrm{GT}}$ mouse embryos might have terminally differentiated into this specific cell type. Embryos arising from heterozygous intercrosses were stained with the trophoblast differentiation marker Troma-1, a trophectoderm-specific monoclonal antibody raised against cytokeratin endo-A (Brulet et al. 1980). At the eight-cell stage, Troma-1 was absent from wild-type embryos but was clearly present in Gmnn ${ }^{\text {GT/GT }}$ embryos and in $>32$-cell embryos containing at least one wildtype Gmnn allele (Fig. 2A,B). In addition, formation of a central blastocyst cavity was evident in some of the eight-cell mutant embryos, suggesting that trophoblasts in Gmnn GT/GT embryos remain functional and engage in fluid transport driven by an intact basal membrane $\mathrm{Na}^{+} /$ $\mathrm{K}^{+}$-ATPase (Fig. 3). Embryos obtained from Gmnn ${ }^{+/ \mathrm{GT}}$ intercrosses were also stained with Oct4 (Pou5f1). Oct4 is a POU domain transcription factor required for main- 


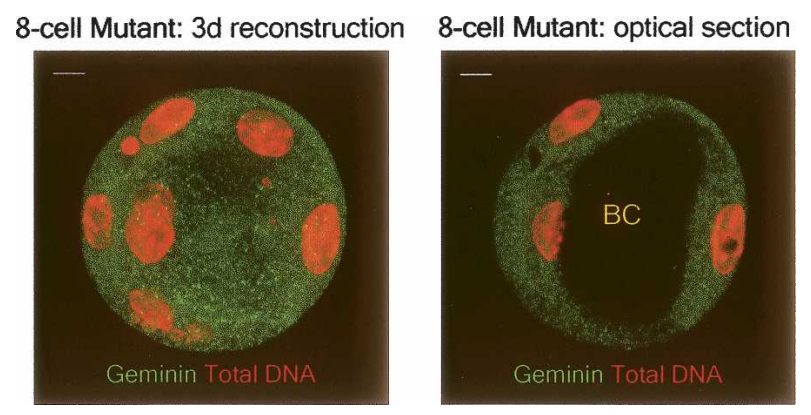

Figure 3. E3.5 geminin-null embryos are capable of forming expanded blastocysts that lack an ICM but contain a blastocoele. (Left) $\mathrm{Z}$ stacks collected through a geminin-null embryo and compiled into a single image show the formation of an expanded blastocyst consisting of eight cells with massively enlarged nuclei. (Red) Total DNA; (green) geminin. (Right) Confocal section through the same embryo shows the presence of a blastocyst cavity (BC). The background in the green channel is deliberately enhanced to illustrate the presence of the blastocoele. Note the absence of nuclear geminin in contrast to wild-type embryos (Figs. 2, 4). Bar, $10 \mu \mathrm{m}$.

tenance of ICM (Nichols et al. 1998). It is expressed in all blastomeres of the cleavage stage embryo and becomes restricted to the pluripotent ICM in the developing blastocyst (Palmieri et al. 1994). Consistent with the TGC differentiation phenotype shown by Troma-1 positivity, Oct4 was absent in all cells of geminin-null but not wildtype mouse embryos (Fig. 4A). All cells in Gmnn ${ }^{\text {GT/GT }}$ mouse embryos therefore lose pluripotency, endoreduplicate, and consist exclusively of terminally differentiated TGCs that are capable of forming eight-cell embryos resembling blastocysts with a blastocoelic cavity but notably lacking ICM.

Our results demonstrate that loss of zygotic geminin promotes trophectoderm formation. The transcription factor Caudal-related homeobox $2(\mathrm{Cdx} 2)$ has recently been shown to be involved in commitment to the trophoblast cell lineage. Cdx2 is expressed in prospective trophectoderm as early as the two-cell stage in mouse embryos (Deb et al. 2006). Cdx2 is expressed in trophoblast stem (TS) cells but not in ES cells (Tanaka et al. 1998; Niwa et al. 2000). Interestingly, TS cells cannot be derived from Cdx2 mutant embryos (Strumpf et al. 2005) while ES cells can (Chawengsaksophak et al. 2004). Moreover, repression of $\mathrm{Cdx} 2$ by Oct 4 prevents trophectoderm differentiation in ES cells (Niwa et al. 2005). However, in geminin-null mouse embryos where all cells commit to the trophoblast cell lineage, we noted that $\mathrm{Cdx} 2$ could be detected in some but not all nuclei (Fig. 4B), suggesting that down-regulation of geminin alone may be sufficient to cause trophoblast cell differentiation independently of $\mathrm{Cdx} 2$. Together with Oct4 and $\mathrm{Cdx} 2$, geminin may possibly act as one of the "selector genes" for ICM and trophectoderm fates that negatively regulate each other to reinforce the segregation of these two cell lineages during mammalian embryonic development (Fig. 4C). Although this interpretation deserves further investigation, we did find that in wild-type mouse embryos, geminin is present in all blastomeres from the two-cell stage to the eight-cell stage and that its expression persists but is not exclusively restricted to ICM at the blastocyst stage (Supplementary Fig. S4).

Acquisition of a high DNA content in geminin-null mouse embryos could be caused by true endoreduplica- tion or local overreplication. The cdk inhibitor $\mathrm{p} 57^{\text {Kip } 2}$ is expressed specifically in rat choriocarcinoma cells during "gap-like" phases of endocycles (Hattori et al. 2000). If the giant cells of geminin-null mouse embryos were indeed undergoing endoreduplication, then we would expect some of them to be in gap phases and to be positive for $\mathrm{p} 57^{\text {Kip2 }}$. Indeed, $\mathrm{p} 57^{\text {Kip2 }}$ was enriched in most nuclei of geminin-null mouse embryos (Supplementary Fig. S5). This supports the idea that geminin-null mouse embryos undergo true endoreduplication, a suggestion that is further supported by the observation presented below that geminin is down-regulated in trophoblast giant cells.

\section{A WTE3.5}
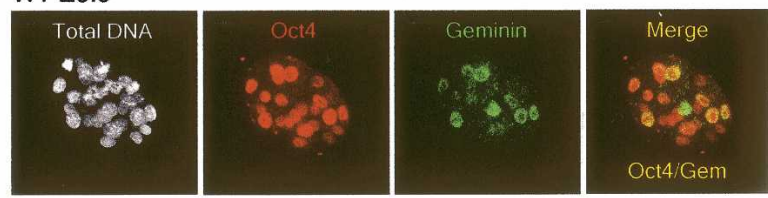

Mutant E3.5
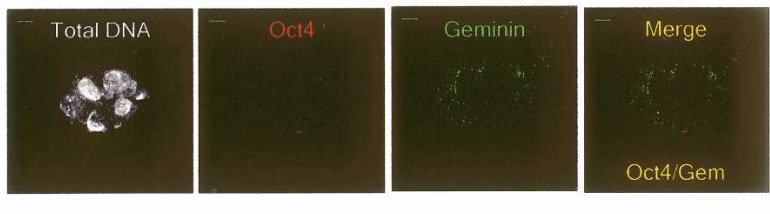

B WTE3.5
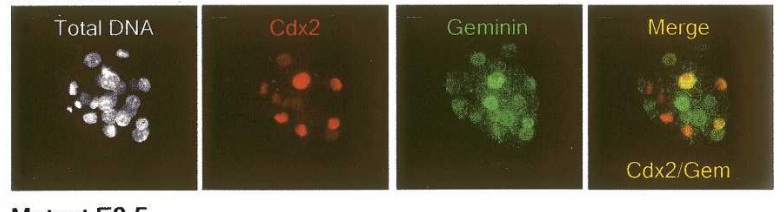

Mutant E3.5
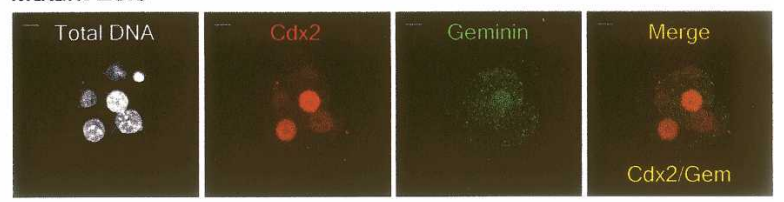

C

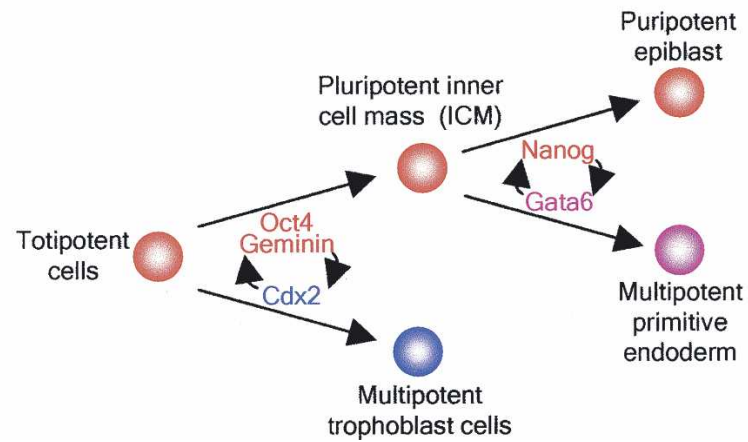

Figure 4. Loss of geminin during mouse embryogenesis results in commitment to the trophectoderm cell lineage independent of Cdx2. (A) The pluripotent cell marker Oct4 (red) is absent in geminin-null mouse embryos. (White) Total DNA; (green) geminin. (B) $\mathrm{Cdx} 2$ (red) is up-regulated in some but not all nuclei of geminin-null mouse embryos. (White) Total DNA; (green) geminin. Bar, $10 \mu \mathrm{m}$. (C) Model for cell lineage determination during early mammalian embryogenesis (adapted from Ralston and Rossant 2005 with permission from Blackwell Publishing (C 2005)|. Segregation of ICM and trophectoderm may be possible through repression of Cdx2 by Oct4/ geminin and repression of Oct4/geminin by Cdx2. Antagonism between Nanog and Gata6 subsequently leads to segregation of epiblast and primitive endoderm within the ICM. 
Since ablation of geminin can trigger premature endoreduplication and TGC formation, we investigated whether geminin is indeed down-regulated in endoreduplicating TGCs of wild-type mouse blastocysts. We cultured E3.5 blastocysts on glass coverslips for 3-5 d to form trophoblast outgrowths and allow for TGC endoreduplication. Geminin levels were then assessed by indirect immunofluorescence. Interestingly, geminin was present at distinctly lower levels in actively replicating TGCs compared with its expression in ICM. Geminin was undetectable in about half of the actively replicating TGCs, whereas it was present in all S-phase cells of the ICM (Fig. 5A). Geminin was absent in nonreplicating TGCs, which include TGCs in the gap-like phases and terminally differentiated TGCs.

The pattern of geminin expression could result from progressive transcriptional shutdown during TGC differentiation or post-translational down-regulation by proteasome-mediated degradation. To distinguish between these possibilities, blastocysts were treated with the proteasome inhibitor MG132 and pulsed with BrdU to detect S-phase cells. Geminin was dramatically stabilized in all S and gap-like phase TGCs (Fig. 5A). As expected, stabilization of geminin in endoreduplicating TGCs still allowed elongation of DNA replication to proceed (McGarry and Kirschner 1998). Since geminin is targeted for degradation by the APC/C in Xenopus embryos and

A

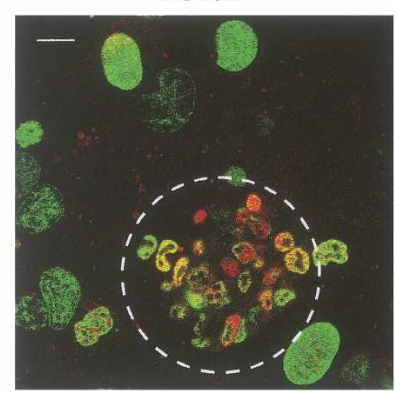

Geminin DNA replication

B

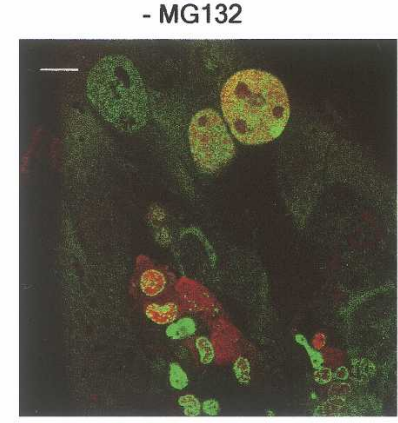

Cyclin A2 DNA replication
+ MG132

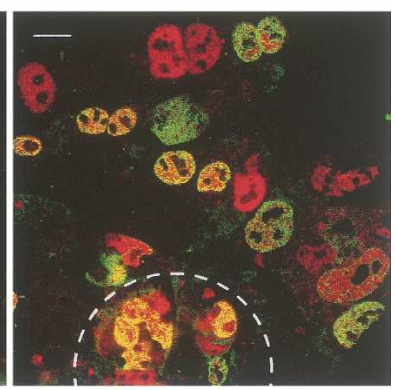

+ MG132

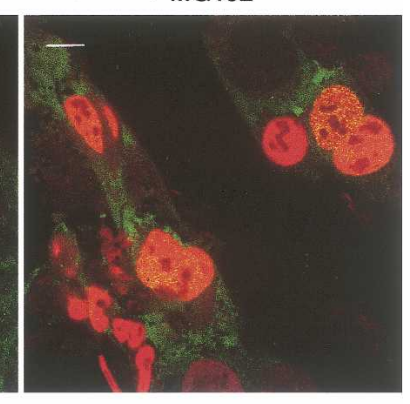

Figure 5. Geminin and cyclin A2 are constitutively degraded in interphase TGCs of wild-type blastocysts. (A) Wild-type blastocysts, cultured on coverlips for 3-5 d to form trophoblast outgrowths, were pulsed with BrdU, fixed, and double-labeled for geminin (red) and BrdU (green). Blastocysts were cultured in the presence or absence of the proteasome inhibitor MG132 for $6 \mathrm{~h}$ prior to BrdU pulse labeling and fixation. The white circles outline the ICM. (B) Wild-type blastocysts were treated as in $A$ and double-labeled for cyclin A2 (red) and BrdU (green). Bar, $50 \mu \mathrm{m}$. mammalian somatic cells (McGarry and Kirschner 1998; Wohlschlegel et al. 2000; Shreeram et al. 2002), the continuous degradation of geminin in interphase TGCs suggests that the APC/C might be constitutively active in these cells. Consistent with this interpretation, the APC/C target cyclin A2 is also stabilized in S and gaplike phases of TGCs by MG132 treatment (Fig. 5B).

There was a striking correlation between geminin down-regulation and endoreduplication in trophoblast outgrowths. We also observed progenitor trophoblast cells in uncultured E3.5 mouse embryos in which some geminin persists (Fig. 2; Supplementary Fig. S4), which might suggest that endoreduplication proper starts at E4.5. Alternatively, geminin present in trophectoderm at E3.5 might be inactivated, possibly by ubiquitination in preparation for degradation, or transiently inactivated to allow pre-RC formation. In spite of this caveat, it is clear that ablation of geminin induces endoreduplication in mouse embryos and that DNA replication in TGCs occurs in the absence of geminin.

In summary, we have shown that suppression of geminin synthesis by genetic ablation can function as the trigger for endoreduplication and trophoblast cell differentiation in uncommitted cells of the early mouse embryo. A high DNA content, presence of the TGC markers Troma-1 and Cdx2, absence of the pluripotency marker Oct4, and formation of a blastocoelic cavity all demonstrate that geminin-deficient embryos consist exclusively of functional "trophoblast-like" giant cells. Further investigations are required to determine whether geminin suppresses endoreduplication and trophoblast differentiation independently in uncommitted cells or if one effect causes the other. For example, geminin may be required for pluripotency by maintaining Oct4 expression and repressing Cdx 2 in ICM cells. It will also be interesting to investigate whether transgenic Cdt1 mouse embryos share the same phenotype.

The observation that geminin and cyclin A2 are continuously degraded in mouse TGCs implies that the $\mathrm{APC} / \mathrm{C}$ is constitutively activated in these cells. In this respect, it is interesting to note that the Drosophila and Medicago (alfalfa) orthologs of the APC/C activator Cdh1 are dispensable for mitotic cycles but are present in endoreduplication-competent cells, in which cyclin A2 is absent (Roudier et al. 2003; Vinardell et al. 2003; Schaeffer et al. 2004). This raises the intriguing possibility that constitutive APC/C activation is a general phenomenon of endoreduplication cycles.

\section{Materials and methods}

\section{$R T-P C R$}

RT-PCR was performed on RNA isolated from ES cell line XT0615 according to the manufacturer's instructions. Two rounds of $5^{\prime}$ rapid amplification of cDNA ends (5' RACE) were carried out using PCR primers 5'-AGTATCGGCCTCAGGAAGATCG-3' and 5'-ATTCAGGCTGCG CAACTGTTGGG-3'. Gene trap insertion into geminin was confirmed by using a primer for geminin exon 1 5'-CGGCGCGTCAGCTACC GGGTCGGCGGGTTA-3' and the first 5' RACE primer designed against the $\beta$-geo fusion, giving a 320-base-pair (bp) product.

\section{Genotyping}

Genotyping was performed by either Southern blotting or PCR. For Southern blotting, a 331-bp geminin probe (5'-AAAATTCCTCAG CTTCAACTTTTTTTGTTAGCTTG-3' and 5'-AGGTCACCGCGGTT CCTCTTGCCTTAG-3') was used on BamHI-digested genomic DNA to give a 19.9-kb fragment for wild-type allele and a 9.4-kb band for the gene 
trap $\left(G m n n^{G T}\right)$ allele. For PCR genotyping, the wild-type allele was identified by amplification of a 406-bp product $\left(5^{\prime}\right.$-CTCTGGTGTTCGC TCTTCCT-3' and 5'-TGTACCTCCTGCCTCAACCT- $\left.3^{\prime}\right)$ and the gene trap allele $\left(G m n n^{G T}\right)$ was identified by amplification of a $507-\mathrm{bp}$ product designed against the $\beta$-geo insert (5'-GTGGCGACGACTCCTGGAGC CCGTCAGTA-3' and 5'-TCAAGCGTATGCAGCCGCCGCATTGCA TCAGCCAT-3').

\section{Immunofluorescence}

Wild-type blastocysts were cultured for 3-5 d on poly-lysine-coated coverslips, and some were treated with $20 \mu \mathrm{M}$ MG132 (CalBiochem) for $6 \mathrm{~h}$ prior to fixation in $4 \%$ paraformaldehyde. Blastocysts were permeabilized in $0.5 \%$ Triton X-100 and stained as previously described for tissue culture cells (Gonzalez et al. 2004). Blastocysts were stained with cyclin A2 (a kind gift from Dr. Mark Carrington, Cambridge, UK), geminin (Gonzalez et al. 2004), Oct4 (BD Biosciences), Cdx2 (Biogenex), phosphohistone H2AX (Ser139) (clone JBW301; Upstate Cell Signaling Solutions), and p57 Kip2 (M-20; Santa Cruz Biotechnology). The Troma-1 monoclonal antibody developed by Rolf Kemler was obtained from the Developmental Studies Hybridoma Bank developed under the auspices of the NICHD and maintained by The University of Iowa (Department of Biological Sciences, Iowa City, IA). Embryos were stained with DAPI, and serial confocal sections ( $\mathrm{Z}$ stacks) were collected at $0.5-\mu \mathrm{m}$ intervals on a Zeiss Axioplan 2 microscope. Relative DNA contents were assessed semiquantitatively as a measure of nuclear volume and fluorescence intensity using Volocity LE 3.0.2 software.

\section{Acknowledgments}

We first thank Dr. Wen-Hann Tan for helping to initiate this collaboration between the MRC Cancer Cell Unit and the Wellcome Trust Sanger Institute. We thank Professor Azim Surani, Dr. Petra Hajkov, Dr. Bill Skarnes, Paul Tesar, and Dr. Tony Mills for helpful suggestions; Sarah Vowler for statistical analysis; Dr. Mark Carrington for the kind gift of cyclin A2 antibody; Pam Stacey for technical assistance; and Si-Qin for microinjections and harvesting wild-type E3.5 embryos. We thank the Medical Research Council, Cancer Research UK, and the Wellcome Trust for funding. M.A.G. is the recipient of an MRC Clinical Research Training Fellowship, K.K.T. and M.H. are the recipients of an MRC Career Development Fellowship, D.J.A. is supported by a Cancer ResearchUK Career Development Award, and L.v.d.W. is recipient of a C.J. Martin Fellowship from the National Health and Medical Research Council of Australia.

\section{References}

Brulet, P., Babinet, C., Kemler, R., and Jacob, F. 1980. Monoclonal antibodies against trophectoderm-specific markers during mouse blastocyst formation. Proc. Nat1. Acad. Sci. 77: 4113-4117.

Chawengsaksophak, K., de Graaff, W., Rossant, J., Deschamps, J., and Beck, F. 2004. Cdx2 is essential for axial elongation in mouse development. Proc. Natl. Acad. Sci. 101: 7641-7645.

Deb, K., Sivaguru, M., Yong, H.Y., and Roberts, R.M. 2006. Cdx2 gene expression and trophectoderm lineage specification in mouse embryos. Science 311: 992-996.

Del Bene, F., Tessmar-Raible, K., and Wittbrodt, J. 2004. Direct interaction of geminin and Six3 in eye development. Nature 427: 745-749.

Geng, Y., Yu, Q., Sicinska, E., Das, M., Schneider, J.E., Bhattacharya, S., Rideout, W.M., Bronson, R.T., Gardner, H., and Sicinski, P. 2003. Cyclin E ablation in the mouse. Cell 114: 431-443.

Gonzalez, M.A., Tachibana, K.K., Chin, S.F., Callagy, G., Madine, M.A., Vowler, S.L., Pinder, S.E., Laskey, R.A., and Coleman, N. 2004. Geminin predicts adverse clinical outcome in breast cancer by reflecting cell-cycle progression. J. Pathol. 204: 121-130.

Hattori, N., Davies, T.C., Anson-Cartwright, L., and Cross, J.C. 2000. Periodic expression of the cyclin-dependent kinase inhibitor p57(Kip2) in trophoblast giant cells defines a G2-like gap phase of the endocycle. Mol. Biol. Cell 11: 1037-1045.

Luo, L., Yang, X., Takihara, Y., Knoetgen, H., and Kessel, M. 2004. The cell-cycle regulator geminin inhibits Hox function through direct and polycomb-mediated interactions. Nature 427: 749-753.

McGarry, T.J. and Kirschner, M.W. 1998. Geminin, an inhibitor of DNA replication, is degraded during mitosis. Cell 93: 1043-1053.

Melixetian, M., Ballabeni, A., Masiero, L., Gasparini, P., Zamponi, R., Bartek, J., Lukas, J., and Helin, K. 2004. Loss of Geminin induces rereplication in the presence of functional p53. J. Cell Biol. 165: 473482.

Nichols, J., Zevnik, B., Anastassiadis, K., Niwa, H., Klewe-Nebenius, D., Chambers, I., Scholer, H., and Smith, A. 1998. Formation of pluripotent stem cells in the mammalian embryo depends on the POU transcription factor Oct4. Cell 95: 379-391.

Niwa, H., Miyazaki, J., and Smith, A.G. 2000. Quantitative expression of Oct-3/4 defines differentiation, dedifferentiation or self-renewal of ES cells. Nat. Genet. 24: 372-376.

Niwa, H., Toyooka, Y., Shimosato, D., Strumpf, D., Takahashi, K., Yagi, R., and Rossant, J. 2005. Interaction between Oct3/4 and Cdx2 determines trophectoderm differentiation. Cell 123: 917-929.

Palmieri, S.L., Peter, W., Hess, H., and Scholer, H.R. 1994. Oct-4 transcription factor is differentially expressed in the mouse embryo during establishment of the first two extraembryonic cell lineages involved in implantation. Dev. Biol. 166: 259-267.

Parisi, T., Beck, A.R., Rougier, N., McNeil, T., Lucian, L., Werb, Z., and Amati, B. 2003. Cyclins E1 and E2 are required for endoreplication in placental trophoblast giant cells. EMBO I. 22: 4794-4803.

Ralston, A. and Rossant, J. 2005. Genetic regulation of stem cell origins in the mouse embryo. Clin. Genet. 68: 106-112.

Roudier, F., Fedorova, E., Lebris, M., Lecomte, P., Gyorgyey, J., Vaubert, D., Horvath, G., Abad, P., Kondorosi, A., and Kondorosi, E. 2003. The Medicago species A2-type cyclin is auxin regulated and involved in meristem formation but dispensable for endoreduplication-associated developmental programs. Plant Physiol. 131: 1091-1103.

Schaeffer, V., Althauser, C., Shcherbata, H.R., Deng, W.M., and RuoholaBaker, H. 2004. Notch-dependent Fizzy-related/Hec1/Cdh1 expression is required for the mitotic-to-endocycle transition in Drosophila follicle cells. Curr. Biol. 14: 630-636.

Seo, S., Herr, A., Lim, J.W., Richardson, G.A., Richardson, H., and Kroll, K.L. 2005. Geminin regulates neuronal differentiation by antagonizing Brg1 activity. Genes \& Dev. 19: 1723-1734.

Shreeram, S., Sparks, A., Lane, D.P., and Blow, J.J. 2002. Cell type-specific responses of human cells to inhibition of replication licensing. Oncogene 21: 6624-6632.

Skarnes, W.C., von Melchner, H., Wurst, W., Hicks, G., Nord, A.S., Cox, T., Young, S.G., Ruiz, P., Soriano, P., Tessier-Lavigne, M., et al. 2004. A public gene trap resource for mouse functional genomics. Nat. Genet. 36: 543-544.

Stead, E., White, J., Faast, R., Conn, S., Goldstone, S., Rathjen, J., Dhingra, U., Rathjen, P., Walker, D., and Dalton, S. 2002. Pluripotent cell division cycles are driven by ectopic Cdk2, cyclin A/E and E2F activities. Oncogene 21: 8320-8333.

Strumpf, D., Mao, C.A., Yamanaka, Y., Ralston, A., Chawengsaksophak, K., Beck, F., and Rossant, J. 2005. Cdx2 is required for correct cell fate specification and differentiation of trophectoderm in the mouse blastocyst. Development 132: 2093-2102.

Tachibana, K.K. and Nigg, E.A. 2006. Geminin regulates multiple steps of the chromosome inheritance cycle. Cell Cycle 5: 151-154.

Tanaka, S., Kunath, T., Hadjantonakis, A.K., Nagy, A., and Rossant, J. 1998. Promotion of trophoblast stem cell proliferation by FGF4. Science 282: 2072-2075.

Vinardell, J.M., Fedorova, E., Cebolla, A., Kevei, Z., Horvath, G., Kelemen, Z., Tarayre, S., Roudier, F., Mergaert, P., Kondorosi, A., et al. 2003. Endoreduplication mediated by the anaphase-promoting complex activator CCS52A is required for symbiotic cell differentiation in Medicago truncatula nodules. Plant Cell 15: 2093-2105.

Wohlschlegel, J.A., Dwyer, B.T., Dhar, S.K., Cvetic, C., Walter, J.C., and Dutta, A. 2000. Inhibition of eukaryotic DNA replication by geminin binding to Cdt1. Science 290: 2309-2312.

Zhu, W., Chen, Y., and Dutta, A. 2004. Rereplication by depletion of geminin is seen regardless of p53 status and activates a G2/M checkpoint. Mol. Cell. Biol. 24: 7140-7150. 


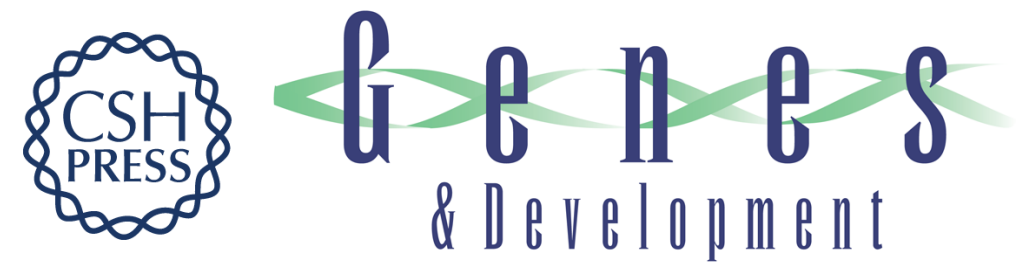

\section{Geminin is essential to prevent endoreduplication and to form pluripotent cells during mammalian development}

Michael A. Gonzalez, Kiku-e K. Tachibana, David J. Adams, et al.

Genes Dev. 2006, 20:

Access the most recent version at doi:10.1101/gad.379706

Supplemental http://genesdev.cshlp.org/content/suppl/2006/06/30/20.14.1880.DC1
Material

References This article cites 28 articles, 13 of which can be accessed free at:

http://genesdev.cshlp.org/content/20/14/1880.full.html\#ref-list-1

License

Email Alerting Receive free email alerts when new articles cite this article - sign up in the box at the top

Service

right corner of the article or click here.

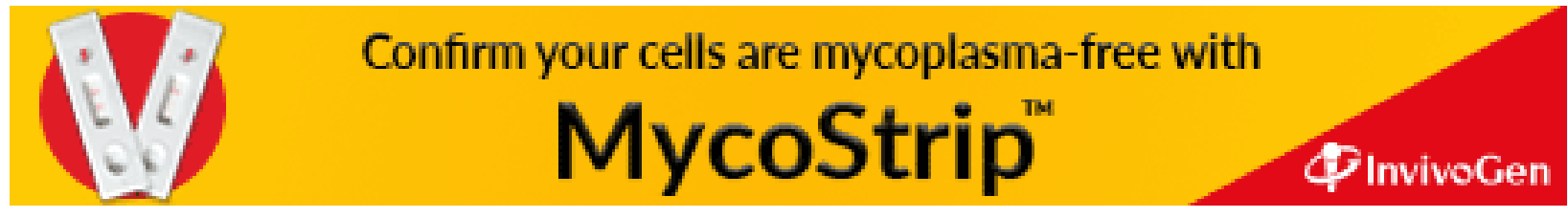

